\title{
تأثير الأساليب الحمائية المقنعة على الصادرات التجارية دراسة حالة قطاع المنتجات البحرية المغرية المبية المراتية
}

\author{
أ محزم حسينة \\ كلية العلوم الاقتصادية و علوم التسبير \\ جامعة قسنطينة 2
}

يـهدف هذا المقال إلى ابراز تأثثير الأساليب الحمائية

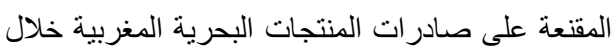

الفترة الممتدة ما بين سنتي 2009 و 2012 ، عن طن طريق المريق

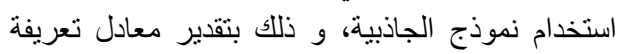



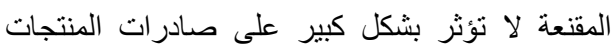

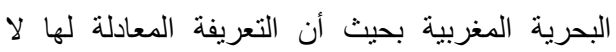

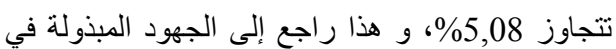

تطوير هذا القطاع بغرض مو مواجهة كل التحديات الحديثة

الكلمات المفتاحية : الأساليب الحمائية المقنعة، معادل

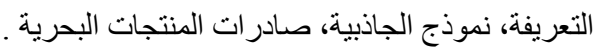

\section{مقدمة: Abstract:}

سعت الدول منذ القدم إلى تطوير

This article aims to highlight the impact of protection is mdisguisedmeasures on the Moroccan marine products exports during the period between the years 2009 and 2012 , by using the gravity model which is estimated the tariffequivalent of disguisedprotectionism. Weconcludethat the protectionismdisguisedmeasuresdoes not gre atly affect the Moroccan marine product exports, because the tariffequivalentdoes not exceed $5.08 \%$, and thisis due to the efforts shown in the development of thissector to copewith all modern challenges.

Keywords:disguisedprotectionismmeasure $\mathrm{s}$, tariffequivalent, gravity model, marine products exports. 
و هنا نجد بأن العديد من الدول استفادة بثكل كبير من حرية التجارة إلا أن بعض قطاعاتهالاقتصادية

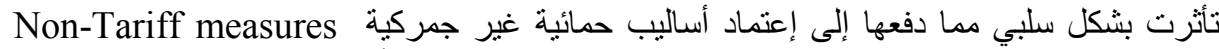

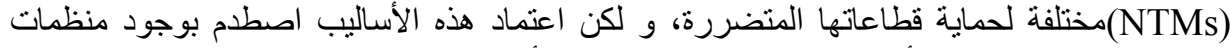

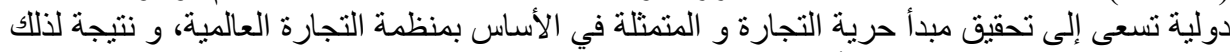

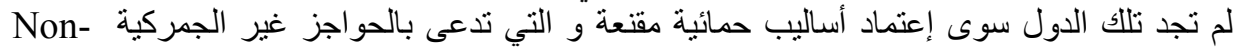
tariff barriers (NTB)

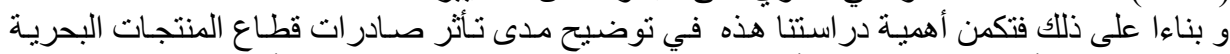

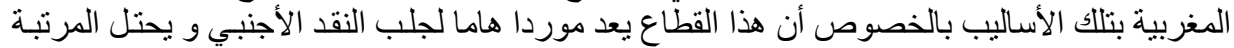

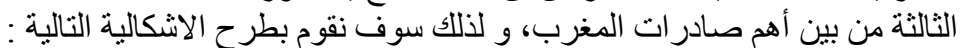



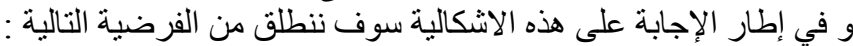



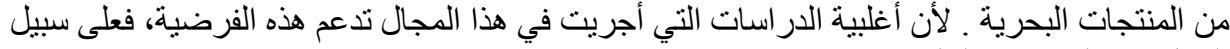

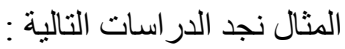

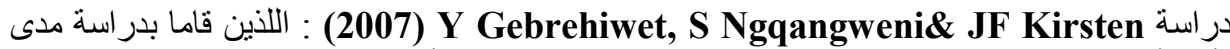

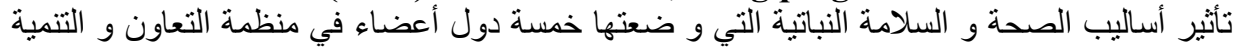
(OECD)



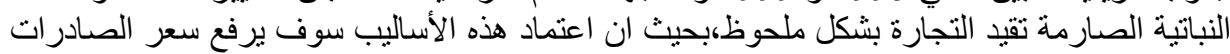

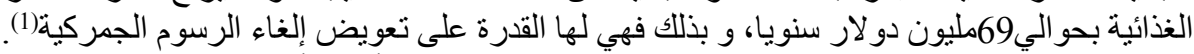

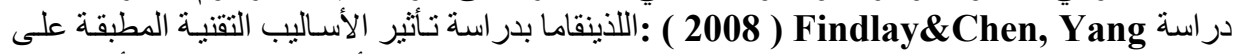



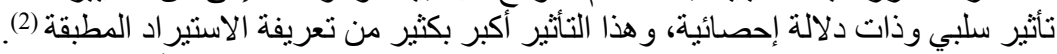

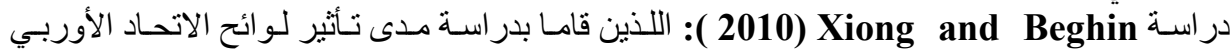



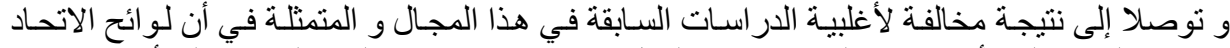

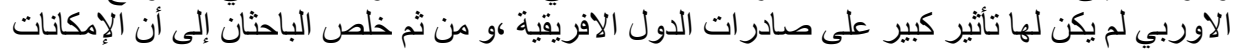

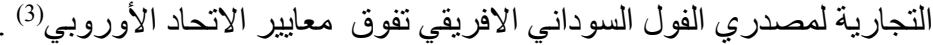

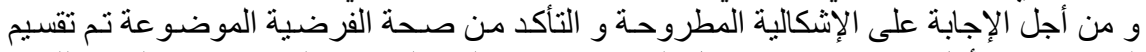

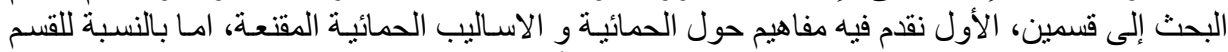

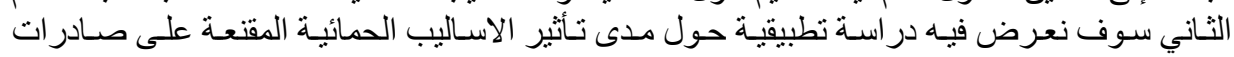

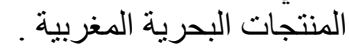

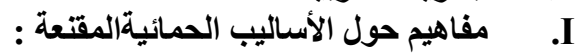

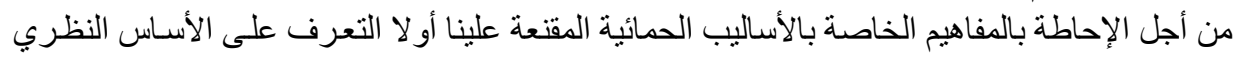



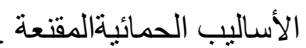

\section{1-1 نظريات الحمائية التقليدية و الحليثة :}

1-1-1 1-1 نظريات الحمائية|لتقليدية :

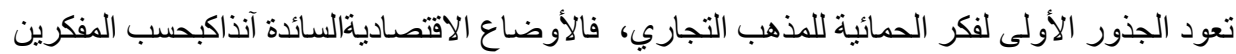



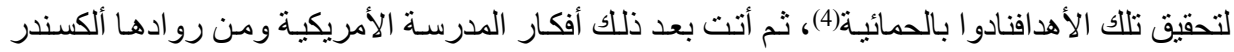


هاملتون " HamiltonAlexander" و الاقتصادي كاري هنري " HenryCarey " الذب قدم نظريـة فئس



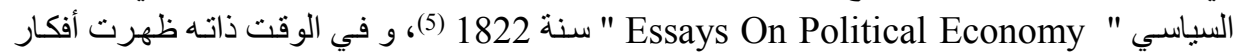
المدرسة الألمانية و ذلك من خلال كتابات فردريك ليست " Friedrich list " حيث قام بتأليف كتاب

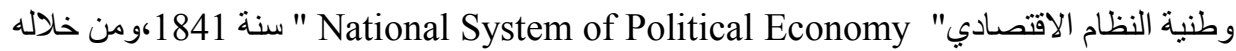



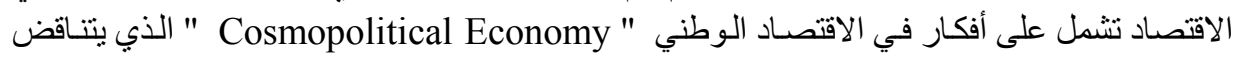

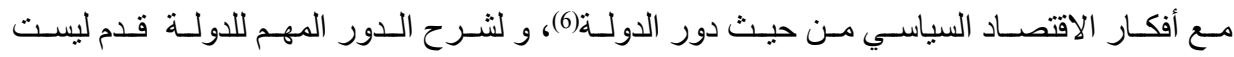

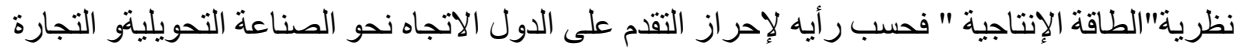

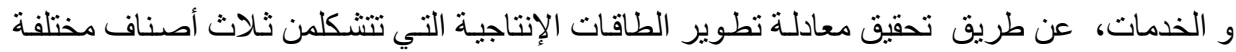



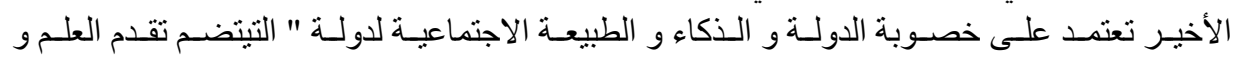

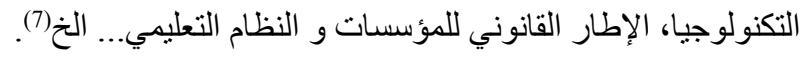

\section{2-1-1 النظريات الحمائية/الحديثة :}

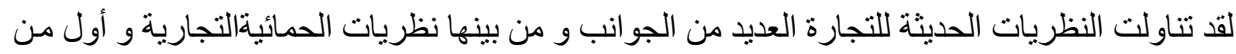

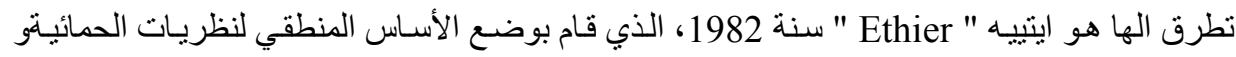

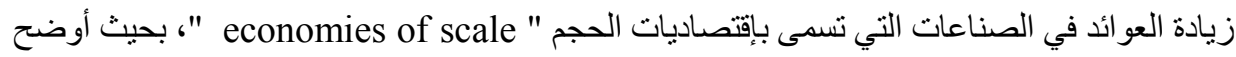

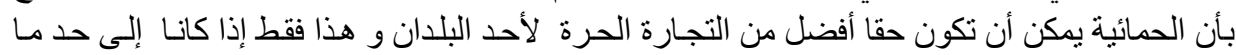

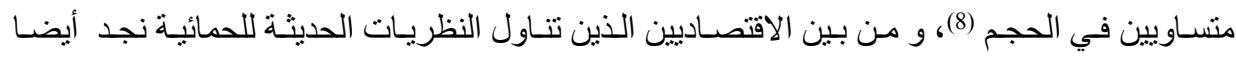

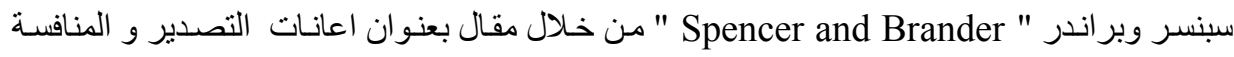

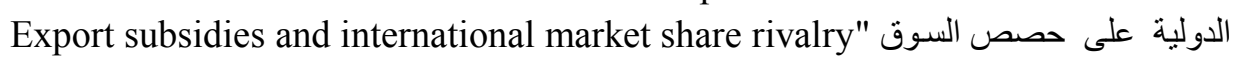



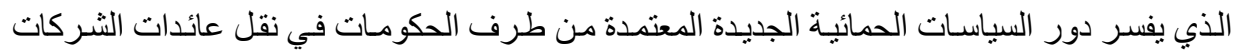



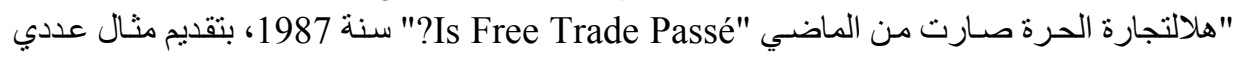



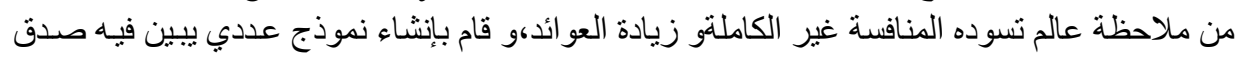
نموذج سياسة التجارة الاستر اتيجية(11).

2-1

هنالك العديد من التعريفات التي أعطيت للسياسات الحمائية، و لعل من أهمها نذكر ما يلي :

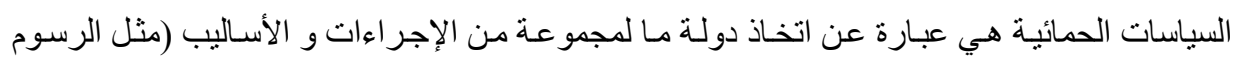

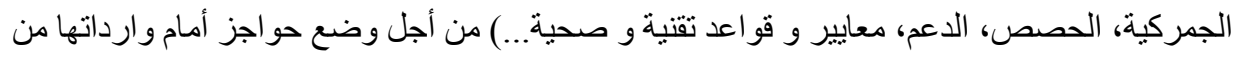
السلع الأجنبية بغية حماية منتوجاتها المحلية من المنافسة الأجنيية (12) . 
كما يمكن تعريفها أيضـا بأنها مجمو عـة الأسـاليب التي تتخذها الحكومة في نطـاق علاقاتها الخارجيـة

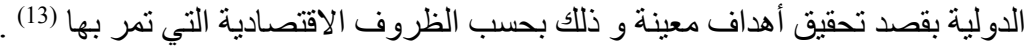



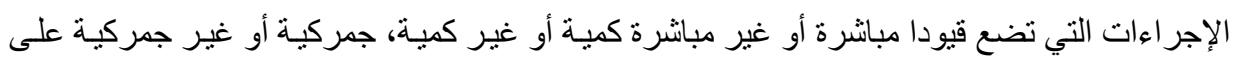
تدفق التجارة الدولية عبر حدود الدولة، لتحقيق أهداف إقتصادية معينة (14).

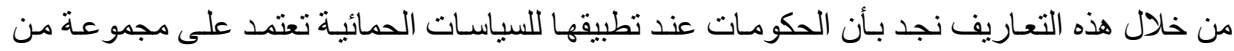



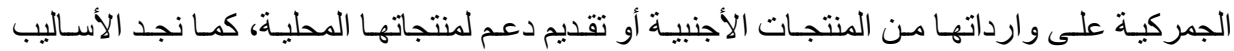

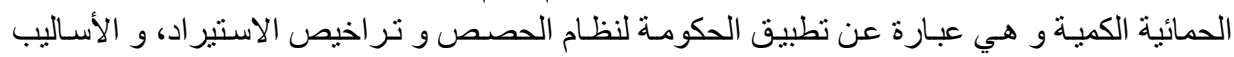

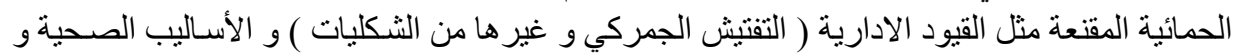

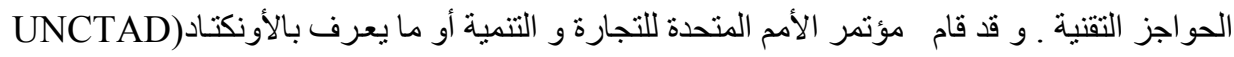



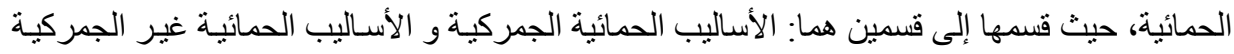

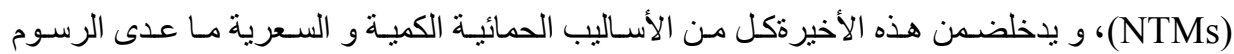

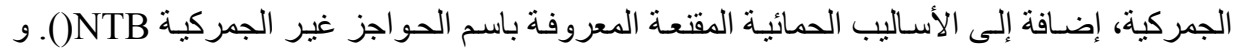

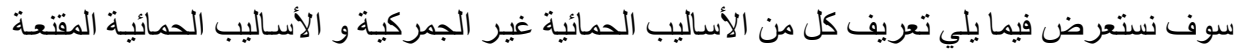

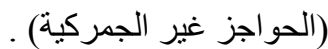

1-2-1 تعريف الأسـاليب الحمائية غير الجمركية (NTMs):في سنة 2010 قدم الانكتاد



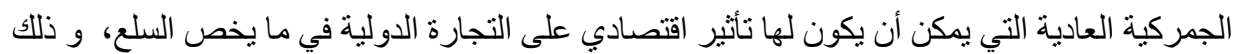
بتغيير الكميات المتداولة أو أسعار ها أو كليهما "(15).

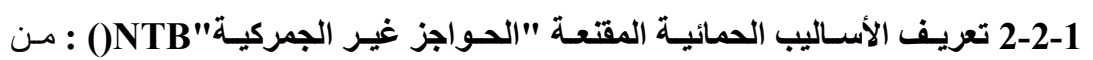

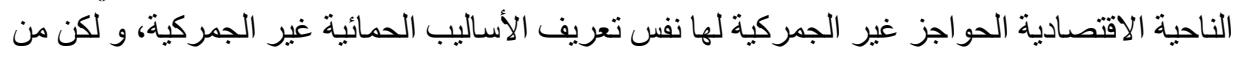

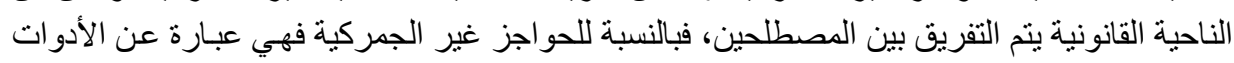

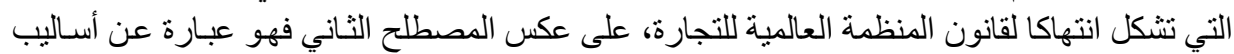

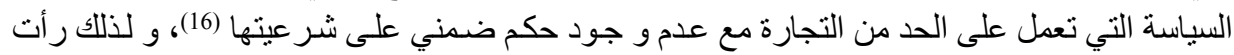

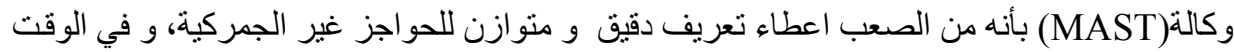

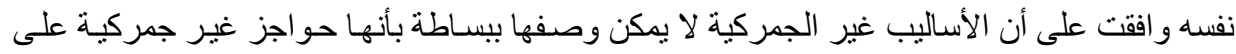



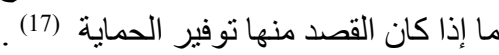
و بناءا على ذلك قامت الاقتصادية سليا دسدير "Célia Disdier"بتعريف الأسـاليب الحمائية المقتعة



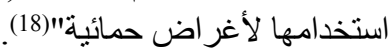

3-1 أنواع الأساليب الحمائيةغير الجمركية و المقتعة :سوف نقام فيما يلي أنواع الأساليب






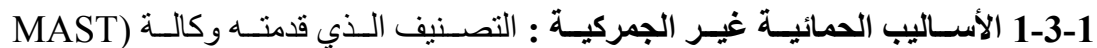

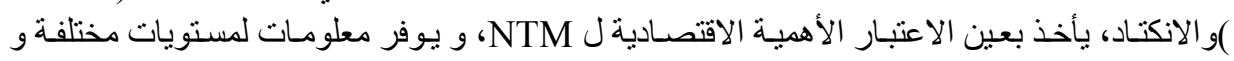







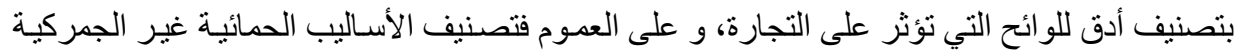
يمكن توضيحها من خلال الجدول التالي (20) : الجدول رقم (1) : تصنيف الأساليب الحمائية غير الجمركية

\begin{tabular}{|c|c|c|}
\hline \multirow[b]{2}{*}{ الواردات } & الأساليب التقنية & 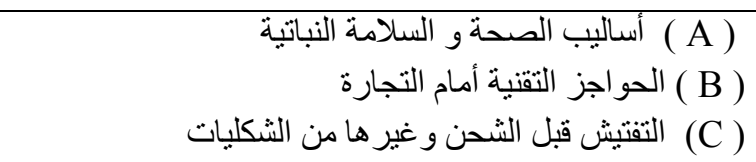 \\
\hline & الأساليب غير & 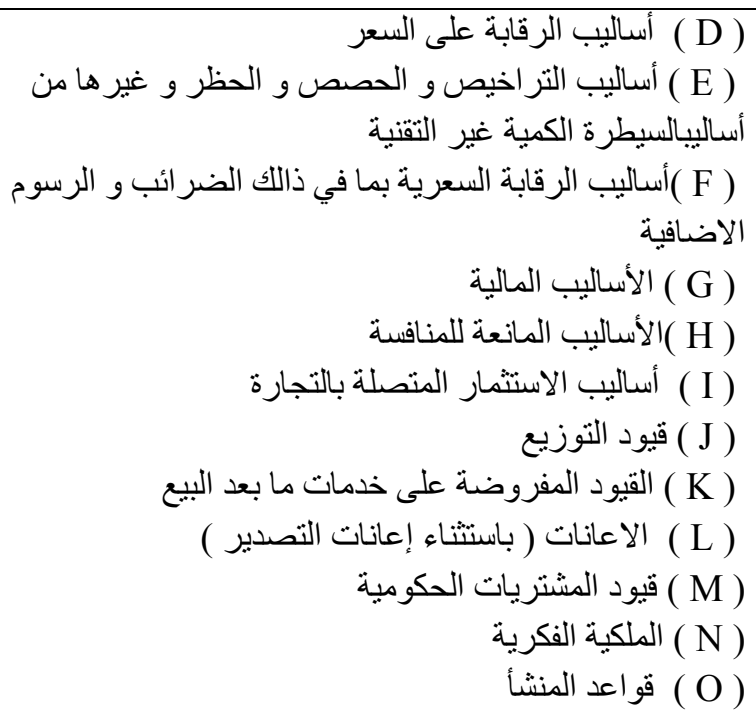 \\
\hline & اسالي & الأساليب المتعلقة بالتصدير \\
\hline
\end{tabular}

Source : UNCTAD, Classification of non-tariff measures,February 2012,p3

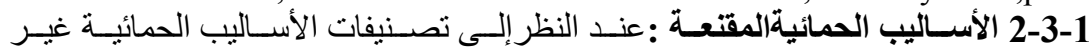

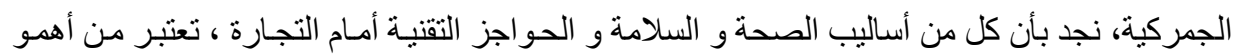

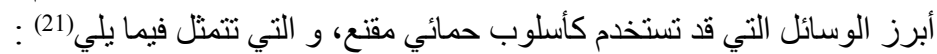





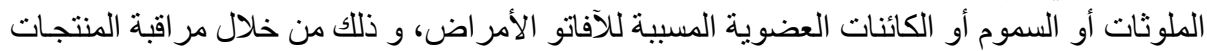

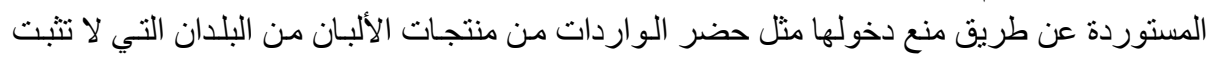


توفرها على ظروف عمل صحية و مرضية، أو وضع بعض التدابير التي يجب الالتزام بها قبل دخول

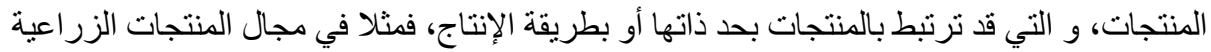



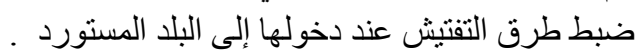

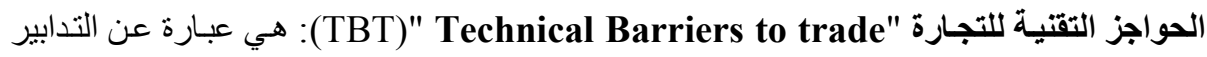

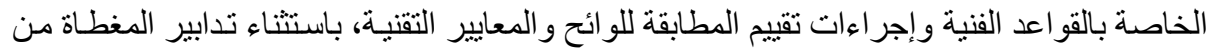

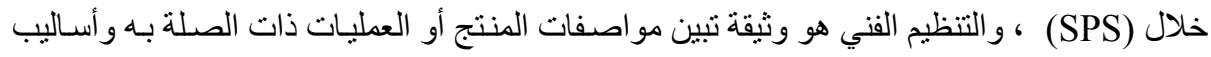

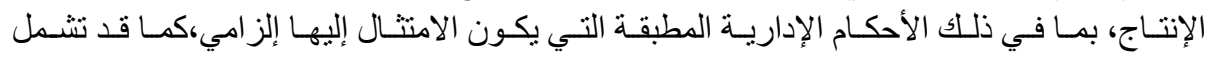



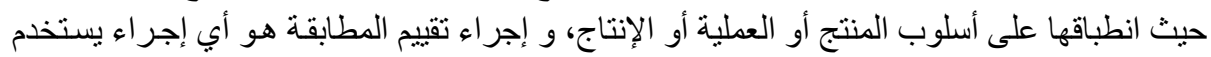





\section{I - I تأثير الأساليب الحمائية المقنعة على قطاع المنتجات البحرية المغربية}

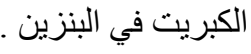

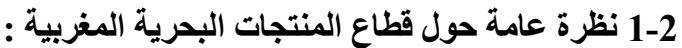

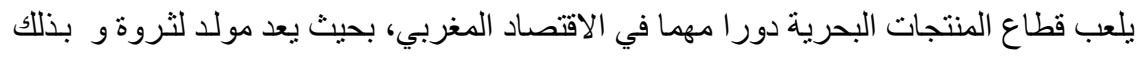



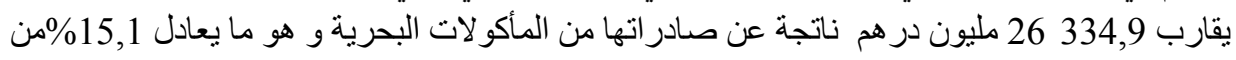





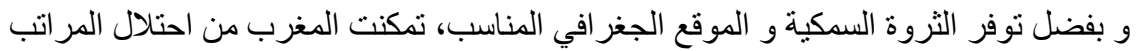
الأولى في انتاج المأكو لات البحرية في إفريقيا .

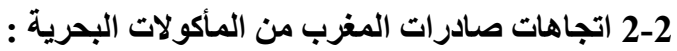



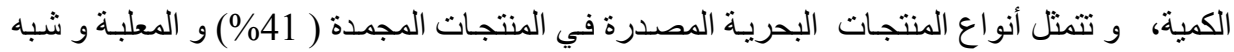

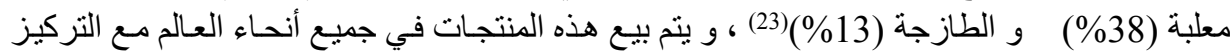



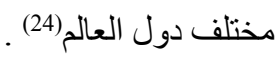

الجدول رقم ( 2) :هيكل الصـادرات المغربيـة من المنتجات البحرية من حيث نوع المنتج و 


\begin{tabular}{|c|c|c|c|c|c|}
\hline الصن اجمالياتي & أمن اجمالي & أسبا ( الصادراتي &  &  & \\
\hline$\% 11$ & $\% 36$ & $\% 56$ & $\% 8$ & $\% 53$ & المجمدة \\
\hline$\% 87$ & $\% 47$ & $\% 25$ & $\% 6$ & $\% 27$ & شبه معلبة و \\
\hline$\% 0$ & $\% 5$ & $\% 10$ & $\% 2$ & $\% 15$ & طازجة \\
\hline$\% 13$ & $\% 12$ & $\% 9$ & $\% 84$ & $\% 5$ & أخرى \\
\hline
\end{tabular}

source :Ministère de l'Agriculture et de la Pêche Maritime, Département de la Pêche Maritime .

من خلال الجدول نستنتج بأن الصادر ات المغربية المتعلقة بالمنتجات البحرية متوجهة بالأسـاس

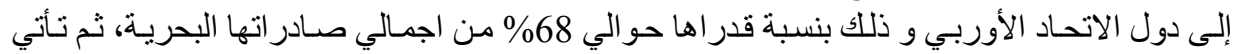

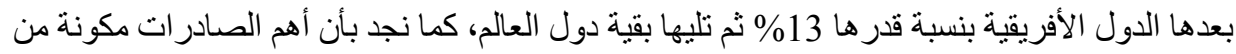



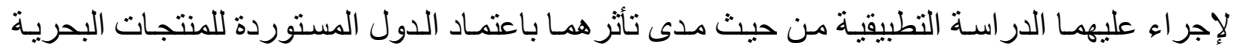
المغربية على المعايير الحمائية المقنعة . لائل

3-2 دراسـة مـدى تـأثير الأسـاليب الحمائيسة المقتعـة على صـادرات المغرب مـن المنتجـات

1-3-2 - 1 النموذج القياسي :

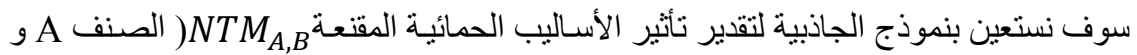

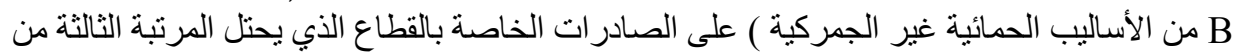

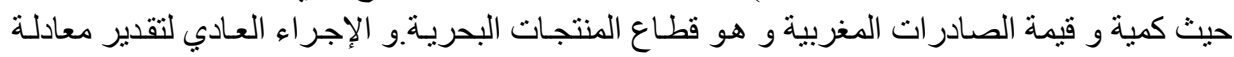


$\ln X_{i j t}=\alpha+\beta_{1} \operatorname{lnGDP} P_{i t}+\beta_{2} \operatorname{lnGDP} P_{j t}+\beta_{3} \ln \left(1+T_{i j t}\right)+\beta_{4} \ln D_{i j}+\beta_{5}$ bord $_{i j}$ $+\beta_{6}$ lang $_{i j}+\beta_{7} \operatorname{col}_{i j}+\beta_{8}$ land $_{i j}+\beta_{9} R T A_{i j}+\beta_{10} N T M$



(US\$ billion) : اللوغاريتم الطبيعي لناتج المحلي الاجمالي للدولة المستوردة lnGDP

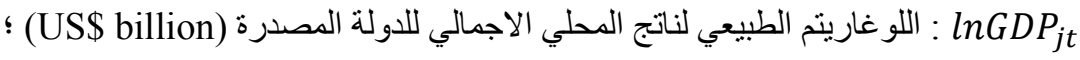
الئا ln $\left(1+T_{i j t}\right)$

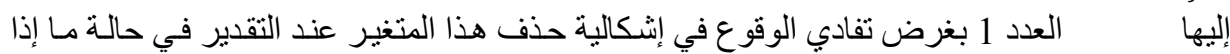




ln $D_{i j}$




المستعرة ، أحد البلدين على الأقل ساحلي ، وجود اتفاقية تجارة حرة بين البلدين ؛

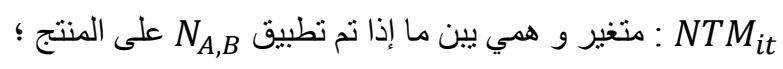
u u l u lijt


بحيث يأخذ هذا المتغير القيمة 1 في حالة تطبيق NTM

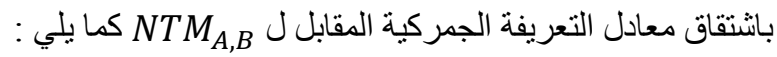

$$
\begin{aligned}
& \ln \widehat{X_{l j t}}=\sum_{n \neq 10}\left(\widehat{\beta_{n}} \Psi_{n t}^{i j}\right)+\widehat{\beta_{10}} N T M_{i t} \\
& \text { عند تعويض قيمة } \\
& \ln X_{\imath J t, N T M}-\ln X_{l J t, n o N T M}=\sum_{n \neq 10}\left(\widehat{\beta_{n}} \Psi_{n t}^{i j}\right)+\widehat{\beta_{10}}(1)-\left(\sum_{n \neq 10}\left(\widehat{\beta_{n}} \Psi_{n t}^{i j}\right)+\widehat{\beta_{10}}(0)\right) \\
& =\widehat{\beta_{10}}
\end{aligned}
$$

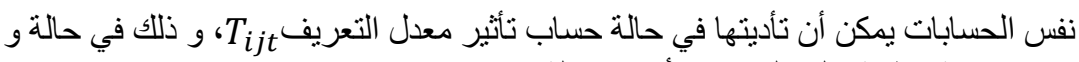

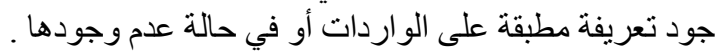

$$
\begin{aligned}
& \ln X_{\iota \jmath t, \operatorname{tar} l f f}-\ln X_{\iota \jmath t, \text { notarlff }}=\sum_{\iota \neq 3}\left(\widehat{\beta}_{\iota} \Psi_{\iota t}^{i j}\right)+\widehat{\beta_{3}}\left(1+T_{i j t}\right)-\left(\sum_{\iota \neq 3}\left(\widehat{\beta}_{\iota} \Psi_{\iota t}^{i j}\right)+\widehat{\beta_{3}}(0)\right) \\
& =\widehat{\boldsymbol{\beta}_{3}} \ln \left(1+T_{i j t}\right)
\end{aligned}
$$

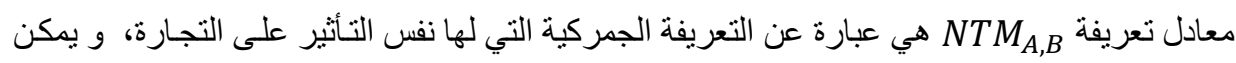

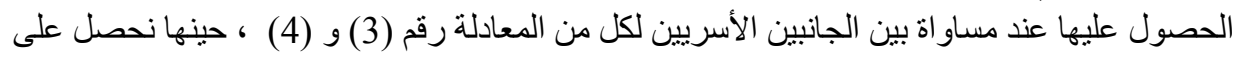

$$
\widehat{\boldsymbol{\beta}}_{10}=\widehat{\boldsymbol{\beta}_{3}} \ln \left(\mathbf{1}+T_{i j t}\right)
$$




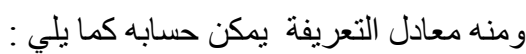

Tariffequivalent $=\exp \left(\widehat{\widehat{\beta_{10}}}\right)-1$

و يتم إجر اء هذه العملية الحسابية بعد تقدير معادلة الجاذبية .

2-3-2 2-2 عينة الاراسة و البيانات:

سوف يتم دراسة مدى نأثير الأساليب الحمائية المقنعة على صادرات المنتجات المغربية ناحية 21 دولية

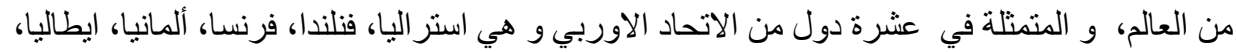

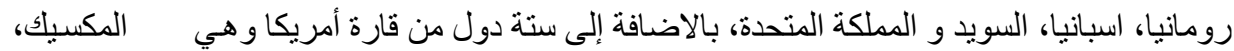

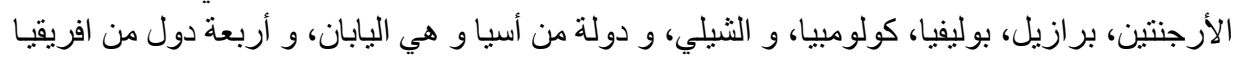

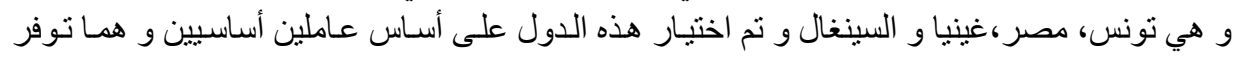

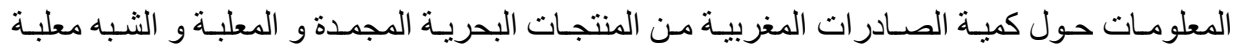
و المعلومات الخاصة بتطبيق أو عدم تطبيق

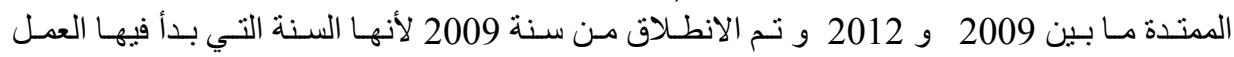



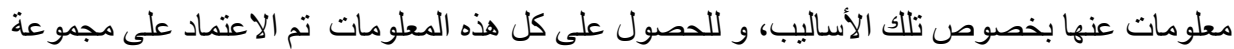

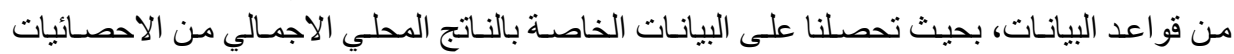

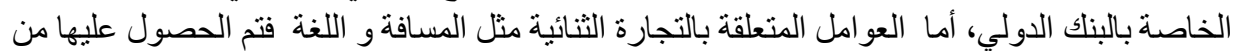




الضر ائب التي تفرضها كل دولة على وارداتها من المنتجات قيد الدراسة :





المصدر : من اعداد الباحثة بالاعتماد على قاعدة بيانات UNCTAD 


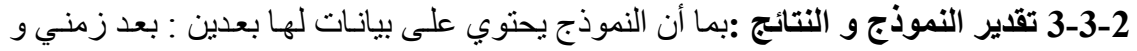


البيانات " panel data" ونتائج التقدير يمكن نوضيحها من خلال الجدول التالي :

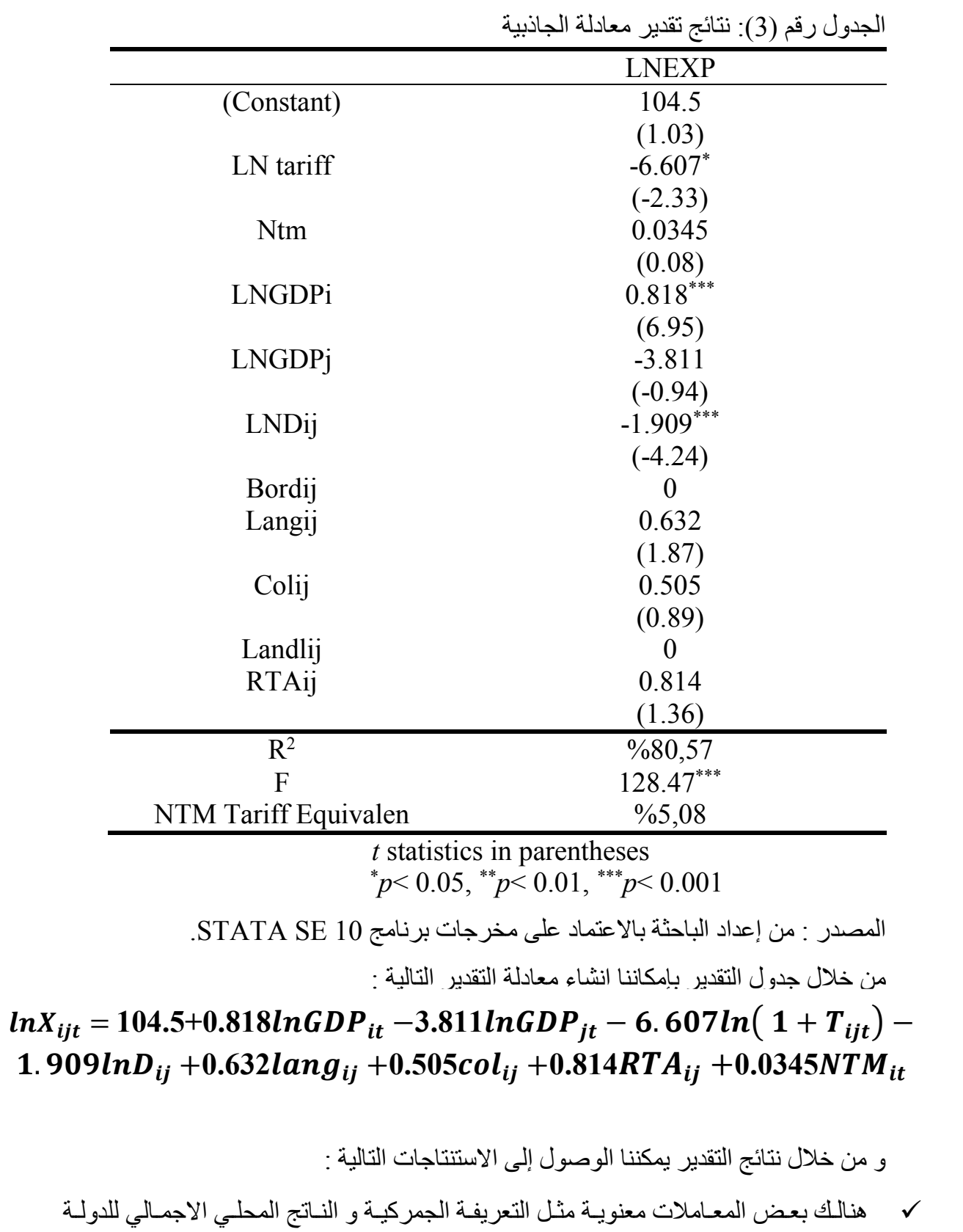


المستوردة، و بعض العوامل أخرى غبر معنوية، إلا أن النموذج ككل معنوي و ذللك بالنظر إلى اختبار

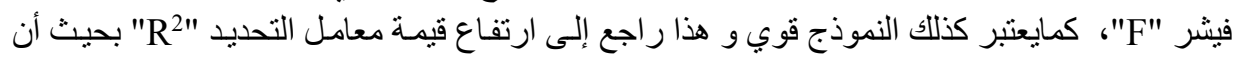

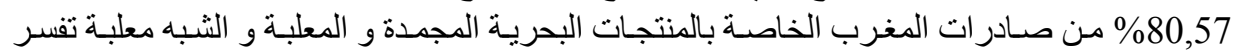
بالمتغير ات المستقلة لهذا النموذج ـ المغرب.

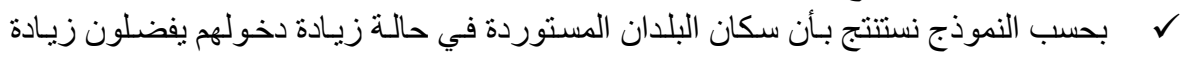

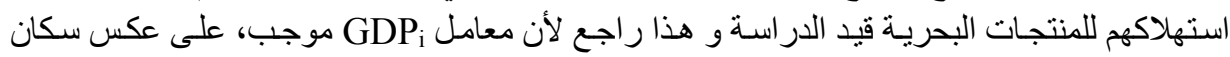

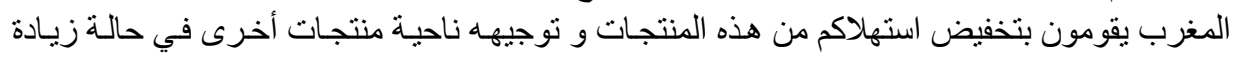

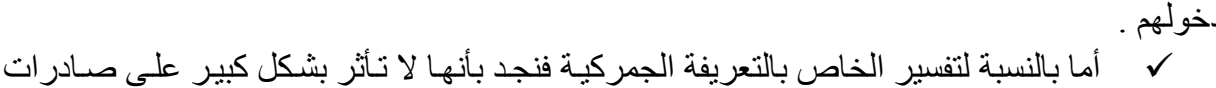

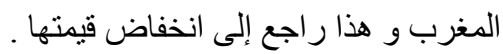



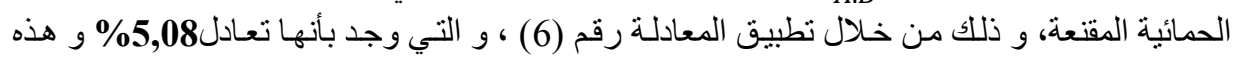



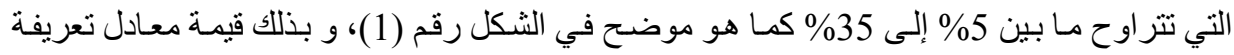



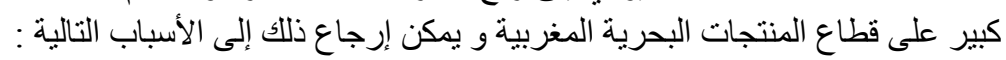

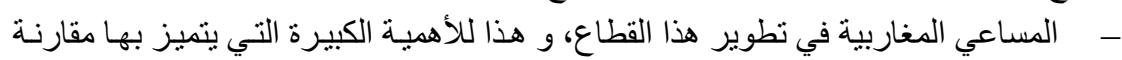

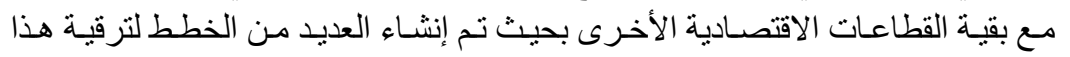

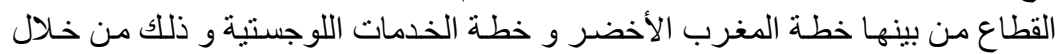



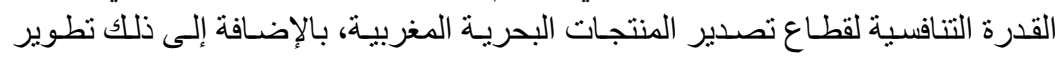



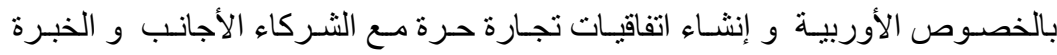

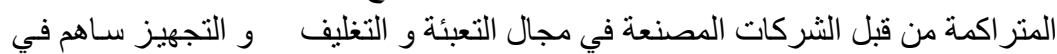

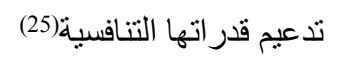

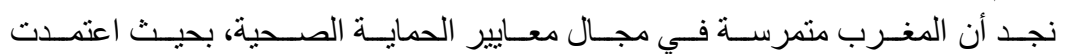

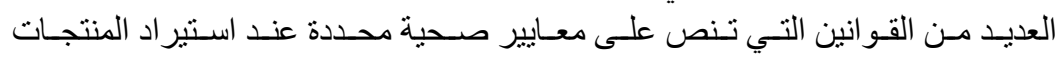



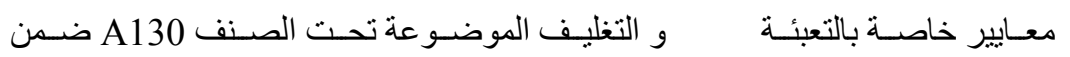

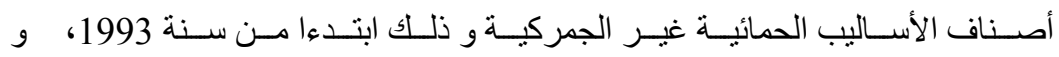

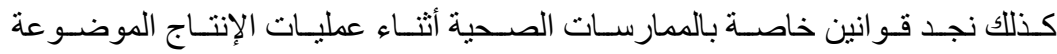

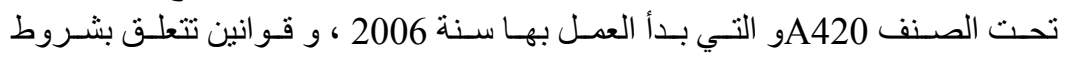


التفتش الموضو عة تحت الصنف A860ابتداء من سنة 1993 ـ 


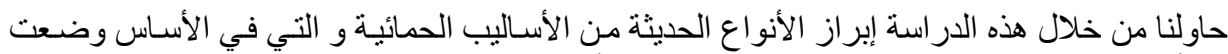

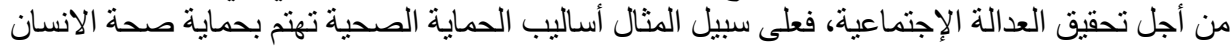



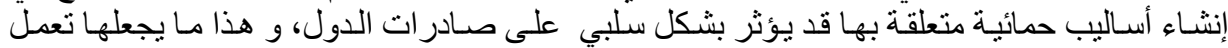

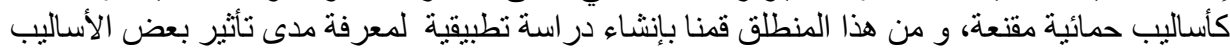



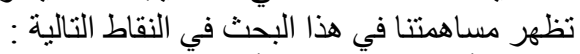



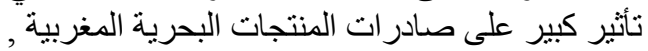

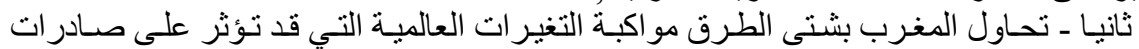

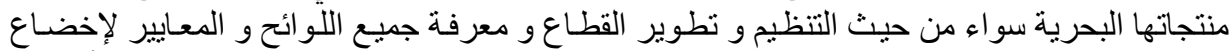

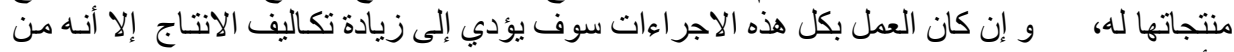

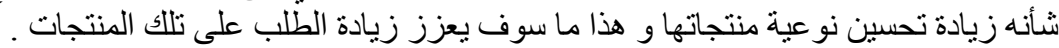



1- Y Gebrehiwet, S Ngqangweni\& JF Kirsten, Quantifying the Trade Effect of Sanitary and Phytosanitary Regulations of OECD Countries on South African Food Exports, Agrekon, Vol 46, No 1, March 2007, p23.

2- Guoxue Wei, Jikun Huang, Jun Yang, The impacts of food safety standards on China's tea exports, China Economic Review,N: 23, 2012,p 253.

3-Xiong, B and Beghin, J C, Aflatoxinredux: does European aflatoxin regulation hurt groundnut exporters from Africa? Annual meeting, Agricultural and Applied Economics Association, 25-27 July, 2010, p1.

4- حسين عمر ، موسوعة الفكر الاقتصـادي ، ج1 ، دار الكتاب الحديث ، القاهرة ، 1994 ، ، ص ص . 160 ، 156 ، 155

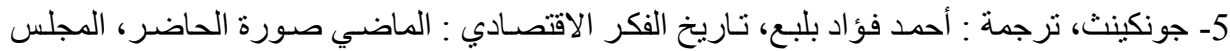

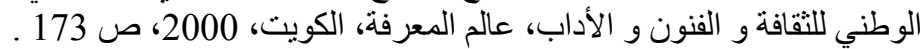

6- MauroBoianovsky, Friedrich List an economic fate of tropical countries,university Brasilia, June 2011,p 4 .

7- Mehdi Shafaeddin, What did Friedrich list actually say ?some clarification on the infant industry argument, UNCTAD, Geneva,july 2000, p 5 .

8-Andrea Maneschi, HOW NEW IS THE "New Trade Theory" of The Past Two Decades?, Vanderbilt University, Working Paper No. 00-W27,July 2000 , p11.

9-james A.Brander, Export subsidies and international market share rivalry, journal of international economics, North-Holland,1985,pp 85-90 .

10-Paul R. Krugman, Is Free Trade Passe?, The Journal of Economic Perspectives, Vol. 1, No. 2, Autumn, 1987, p135.

11-Paul R. Krugman,op.cit,p 136 .

12- Abdelmalki L., Sandretto R., Politiques Commerciales des Grandes Puissances: la tentation du neoprotectionnisme, de boeck, Paris, 2009, p13. 
13- مقيس أسعد عبد المالك، الاتجاهات الحديثة في الاقتصـاد الدولي، دار المعارف، مصر، الطبعة 3،

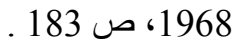

14- عبد المطلب عبد الحميد، السياسات الاقتصادية : تحليل كلي، سلسلة الدر اسـات الاقتصـادية، الطبعـة



15- United Nations Conference on Trade and Development, Non-tariff measures to trade: Economic and Policy Issues for Developing Countries , developing countries in international trade studies, united nations publication , 2013 , p 2 . unctad.org/en/PublicationsLibrary/ditctab20121_en.pdf 19/12/2013 $04: 15$

16- Eric Burgeat, Gérard Viatte, Agricultural Policies in Emerging and Transition Economies 2001, Special Focus On Non-Tariff Measures, Organization For Economic Co-operation And Development (OECD ), France ,2001, PP $20,21$.

17- SudipRanjanBasu,HiroakiKuwahara, Fabien Dumesnil, Evolution of nontariff measures: emerging cases from selected developing countries , policy issues in international trade and commodities study series No. 52 , UNCTAD, Geneva, 2012 , p 8 .

18- Anne-Célia Disdier, Les normes réglementaires, un protectionnisme déguisé ?, la vie des idées, 2 décembre 2009, P 1 . http://www.laviedesidees.fr/IMG/pdf/20091202_disdier.pdf 08/3/2014 15:51

19- United Nations Conference on Trade and Development,Classification

$$
\text { of non-tariff measures,February 2012, }
$$

p2.http://unctad.org/en/PublicationsLibrary/ditctab20122_en.pdf 30/6/2014 08:04

20- United Nations Conference on Trade and Development,op.cit, p3 .

21- United Nations Conference on Trade And Development ,Non-tariff Measures: Evidence from Selected Developing Countries and Future Research Agenda, developing countries in international trade studies , Geneva,2010 ,pp $119-127$.

22- Office des changes, Royaume Du Maroc, Commerce extérieur du Maroc, édition provisoire 2011, p 37 .

23-Direction des Etudes et des Prévisions Financières, Performances des exportations marocaines des produits de la mer sur le marché mondial, Avril $2012, \mathrm{p} 10$.

24- Ministère de l'Agriculture et de la Pêche Maritime, Département de la Pêche Maritime,HALIEUTISSstratégie de développement et de compétitivité du secteur halieutique marocain à l'horizon 2020, p11 .

25- Direction des Etudes et des Prévisions Financières, op.cit. pp 5, 41, 42 .

-Décret $n^{\circ} 2-89-597$ du 25 rebia II 1414 ( 12 octobre 1993 ) pris pour 62 l'application de la loi $\mathrm{n}^{\circ}$ 24-89 édictant des mesures de police sanitaire 
vétérinaire à l'importation d'animaux, de denrées animales, de produits d'origine animale, de produits de multiplication animale et de produits de la mer et d'eau douce, BO $\mathrm{N}^{\circ} 4227$ du 3-11-1993 .http://www.mce.gov.ma/Ministere/pdf/decret\%202-89-59749:\%20sanitairev\%C3\%A9t\%C3\%A9rinaire.pdf $\quad 21 / 9 / 2014 \quad 15$ 